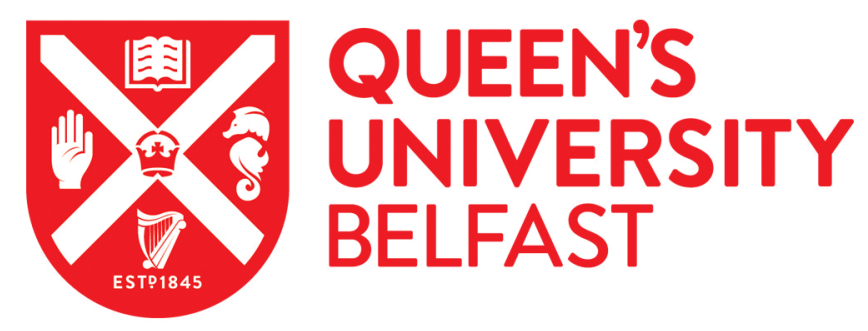

\title{
External validation of a model to determine risk of progression of Barrett's oesophagus to neoplasia
}

Kunzmann, A. T., Thrift, A. P., Johnston, B. T., McManus, D. T., Gavin, A. T., Turkington, R. C., \& Coleman, H. G. (2019). External validation of a model to determine risk of progression of Barrett's oesophagus to neoplasia. Alimentary Pharmacology and Therapeutics, 49(10), 1274-1281. https://doi.org/10.1111/apt.15235

Published in:

Alimentary Pharmacology and Therapeutics

Document Version:

Peer reviewed version

Queen's University Belfast - Research Portal:

Link to publication record in Queen's University Belfast Research Portal

Publisher rights

(C) 2019 John Wiley \& Sons Ltd. This work is made available online in accordance with the publisher's policies. Please refer to any applicable terms of use of the publisher.

\section{General rights}

Copyright for the publications made accessible via the Queen's University Belfast Research Portal is retained by the author(s) and / or other copyright owners and it is a condition of accessing these publications that users recognise and abide by the legal requirements associated with these rights.

Take down policy

The Research Portal is Queen's institutional repository that provides access to Queen's research output. Every effort has been made to ensure that content in the Research Portal does not infringe any person's rights, or applicable UK laws. If you discover content in the Research Portal that you believe breaches copyright or violates any law, please contact openaccess@qub.ac.uk. 
External validation of a Model to Determine Risk of Progression of Barrett's Oesophagus to Neoplasia

Running head: Predicting progression from Barrett's

Andrew T Kunzmann ${ }^{1}$, Aaron P Thrift $* 2,3$, Brian T Johnston ${ }^{4}$, Damian T McManus ${ }^{5}$, Anna T Gavin $^{6}$, Richard C Turkington 7,8 \& Helen G Coleman ${ }^{1,7}$ * Denotes joint first author

Authors' affiliations:

${ }^{1}$ Cancer Epidemiology Research Group, Centre for Public Health, Queen's University Belfast, Belfast, Northern Ireland, United Kingdom

${ }^{2}$ Section of Epidemiology and Population Sciences, Department of Medicine, Baylor College of Medicine, Houston, Texas

${ }^{3}$ Dan L Duncan Comprehensive Cancer Centre, Baylor College of Medicine, Houston, Texas

${ }^{4}$ Royal Victoria Hospital, Belfast Health \& Social Care Trust, Belfast, N. Ireland, United Kingdom

${ }^{5}$ Department of Pathology, Belfast Health \& Social Care Trust, Belfast, N. Ireland, United Kingdom

${ }^{6}$ Northern Ireland Cancer Registry, Queen's University Belfast, Belfast, Northern Ireland, United Kingdom

${ }^{7}$ Centre for Cancer Research and Cell Biology, Queen's University Belfast, Belfast, Northern Ireland, United Kingdom

${ }^{8}$ Belfast City Hospital, Belfast Health \& Social Care Trust, Belfast, N. Ireland, United Kingdom Corresponding author: Dr Andrew Kunzmann, Institute of Clinical Sciences Block B, Queen's University Belfast, Royal Victoria Hospital, Belfast, Northern Ireland, BT12 6BA.

Phone: +44 (0) 2890635009

Fax: $+44(0) 2890235900$

Email: a.kunzmann@qub.ac.uk

Word count: 2973 


\section{Conflicts of interest: None}

\section{Author statement}

Guarantor of article: Dr Andrew Kunzmann

Specific author contributions: Specific author contributions: Study concept and design: APT, ATK \& HGC; acquisition of data: HGC; analysis and interpretation of data: ATK, APT \& HGC; drafting of the manuscript: ATK \& APT; critical revision of the manuscript for important intellectual content: HGC, BTJ, DTM, BTJ, ACG, RCT; statistical analysis: ATK \& APT; obtained funding: APT; study supervision: APT \& HGC.

All authors approved the final version of the article, including the authorship list.

Additional Authorship Options. Andrew T Kunzmann \& Aaron P Thrift should be considered joint first authors.

\section{Acknowledgements}

We express our appreciation to the late Professor Liam Murray, whose contribution to the development of the Northern Ireland Barrett's register and to the field of cancer epidemiology was of great significance. We would also like to acknowledge the contribution of the Tumour Verification Officers in the Northern Ireland Cancer Registry, namely Mrs Kate Donnelly, Mrs Rosemary Ward and Ms Olwyn Dawson, who collected the data presented in this report. This work uses data provided by patients and collected by healthcare services as part of their care and support.

The Northern Ireland Barrett's register was funded by the UK Medical Research Council, Cancer Focus Northern Ireland (formerly the Ulster Cancer Foundation), NI HSC R\&D Office, and Cancer Research UK. The Northern Ireland Cancer Registry is funded by the Public Health Agency. 


\section{SUMMARY}

Background: A risk prediction model containing sex, smoking history, Barrett's oesophagus length and presence of low-grade dysplasia was found to identify individuals at a higher risk of progression to oesophageal adenocarcinoma or high-grade dysplasia.

Aims: To externally validate the model predicting risk of progression from Barrett's oesophagus to neoplasia and assess the predictive utility of additional factors.

Methods: We conducted a retrospective cohort study among individuals from the populationbased Northern Ireland Barrett's register with a histologically confirmed diagnosis of Barrett's oesophagus (with intestinal metaplasia) between 1993 and 2005. The association between a points based model and risk of progression to high-grade dysplasia or oesophageal adenocarcinoma until 2010 was assessed using Cox Proportional Hazards model. Model performance was assessed using area under the receiver operating characteristics curves (AUROC), sensitivity and specificity.

Results: We identified 1,198 individuals with Barrett's oesophagus of whom 54 progressed. The model discriminated reasonably well between progressors and non-progressors, with an AUROC of 0.70 (95\% Cl 0.63-0.78). When categorised into low, intermediate and high risk groups, the AUROC was 0.68 (95\% Cl 0.61-0.74). Compared to using data on dysplasia and segment length for risk stratification, the model resulted in a net reclassification improvement of $20.9 \%$.

Conclusions: This external validation provides further evidence that a model based on sex, smoking, Barrett's segment length and baseline low-grade dysplasia may help to risk stratify patients after an initial diagnosis of Barrett's oesophagus. The model also performed better than the use of low-grade dysplasia status alone for risk-stratification.

Keywords: Barrett's oesophagus; endoscopic surveillance; risk stratification; oesophageal adenocarcinoma 


\section{INTRODUCTION}

Barrett's oesophagus is an established pre-malignant condition of the oesophagus that increases risk of oesophageal adenocarcinoma and dysplasia.

Endoscopic surveillance is often recommended to aid early diagnosis and treatment for dysplasia or cancer [1,2]. Currently, the American Gastroenterology Association guidelines [1] use dysplasia status to determine recommended surveillance interval lengths or treatment, with recommended intervals of 3 to 5 years for individuals with non-dysplastic Barrett's oesophagus, and treatment with Radiofrequency ablation (RFA), Endoscopic mucosal resection (EMR) or surveillance intervals of 12 months recommended for individuals with low-grade dysplasia. The British Society of Gastroenterology (BSG) guidelines [2] are similar, but use Barrett's segment length to determine recommended surveillance interval length in patients with non-dysplastic Barrett's oesophagus, with recommended intervals of 3 to 5 years for short segment Barrett's with specialized intestinal metaplasia $(<3 \mathrm{~cm}), 2$ to 3 years for long segment Barrett's $(\geq 3 \mathrm{~cm})$ and 6 months for Barrett's with low-grade dysplasia. However, endoscopy resources are scarce and the cost-effectiveness of endoscopic surveillance for patients with non-dysplastic Barrett's oesophagus has been questioned [3]. Methods to stratify patients based on risk of progression to oesophageal adenocarcinoma or high-grade dysplasia may help to optimise use of endoscopic resources.

An alternative approach is screening using a risk prediction model. Providing clinicians with a tool that allows them to estimate a patients' risk may better aid them in deciding screening intervals and make future resource utilization even more efficient. Parasa et al [4] used data from 5 centres in the United States (US) and 1 Centre in the Netherlands to develop and internally validate a model to predict progression to oesophageal adenocarcinoma or highgrade dysplasia after an initial diagnosis of Barrett's oesophagus. A model including sex, 
smoking history (ever versus never), Barrett's oesophagus length $(\mathrm{cm})$ and low-grade dysplasia status, had reasonable discriminatory ability, with an area under the receiver operating characteristic curve of 0.76 (95\% Confidence interval [Cl], 0.72-0.80). However, this prediction tool needs to be validated in an independent population before its use can be recommended in clinical practice.[5] To our knowledge, no external validation has been reported to date.

Using data from the Northern Ireland Barrett's register, we aimed to externally validate the model derived by Parasa et al [4] (including sex, smoking history (ever versus never), Barrett's oesophagus length and low-grade dysplasia status). We also examined whether additional factors not examined in the derivation study may help to improve discriminatory performance.

\section{METHODS}

\section{Study population}

The Northern Ireland Barrett's register is a population-based register of all adults diagnosed with columnar-lined epithelium of the oesophagus in Northern Ireland since 1993. The methods of the register formation have been previously described [6]. Briefly, the register was created by reviewing pathology reports of all oesophageal biopsies (corresponding to SNOMED2 codes T62* and SNOMED3 codes T56*) logged in Northern Ireland. A standardised set of guidelines was used for creation of the register, and any pathology reports diagnosing histologically confirmed columnar epithelium of the oesophagus, or synonym terms, were deemed to be Barrett's oesophagus. Reports relating to cancer or confined to the oesophagogastric junction were excluded.

A review of hospital notes of the BE patients diagnosed between 1993 and 2005 was conducted by 1 of 3 trained data abstractors, between March 2005 and March 2010, as previously described $[7,8]$. A standardized electronic proforma was used to extract data on a variety of 
demographic and clinical aspects, including age, sex, Barrett's segment length, body mass index, smoking status, dysplasia status, ulcerative erosion, gastroesophageal reflux symptoms, weight loss and treatment regimen, corresponding to the patient's initial (index) diagnosis of Barrett's oesophagus.

Ethical approval for the Northern Ireland Barrett's register, NICR and related research has been granted by the regional ethics committee of Northern Ireland (15/NI/0203).

\section{Outcomes}

Data from subsequent endoscopic biopsies and linkage to the Northern Ireland Cancer Registry (NICR) was conducted to identify Barrett's oesophagus patients who subsequently developed high-grade dysplasia or oesophageal cancer. The NICR contains data on all patients diagnosed with cancer in Northern Ireland since 1993. The NICR uses the International Classification of diseases V.10 (ICD10) to classify patient tumor type and location based on accompanying clinical information. ICD10 codes were used to identify patients from the NICR with adenocarcinoma or histologically unspecified tumors of the oesophagus. Histologically unspecified tumors were included to ensure complete ascertainment of tumors that may have arisen in patients with Barrett's oesophagus. For the purposes of this analysis, participants were followed up until the first diagnosis of high-grade dysplasia or oesophageal adenocarcinoma, death, or $31^{\text {st }}$ December 2010.

\section{Inclusion and exclusion}

We limited the sample to individuals with Barrett's oesophagus, defined as specialized intestinal metaplasia at biopsy and visible Barrett's segment on endoscopy, who were diagnosed between 1993 and $2005(n=3,148)$ and were included in our hospital case note review sample described above. Multiple endoscopies conducted within 12 months may reflect 
uncertain diagnoses after the initial endoscopy, so individuals who were diagnosed with oesophageal adenocarcinoma or high-grade dysplasia within 12 months of Barrett's oesophagus diagnosis were excluded $(n=58)$. Individuals without complete data on Barrett's segment length, smoking status and dysplasia status were excluded from the primary analyses ( $n=1,950$, Figure 1). Therefore, a total of 1,198 individuals were eligible for inclusion and were included in the primary analysis (Figure 1).

\section{Statistical analysis}

The categorical variables were summarized as counts and percentages. The points based model developed by Parasa et al [4], was used to assign points to each study participant based on male sex (9 points, females: 0 points), ever smoking (5 points), Barrett's segment length (1 point per $\mathrm{cm}$ until $10 \mathrm{~cm}$ ) and presence of low-grade dysplasia at baseline (11 points).

Follow-up time was calculated from 12 months after index date until end of follow-up. We used Cox Proportional hazards models to assess the association between the points based model (including the assigned points on a continuous scale) and risk of progression to oesophageal adenocarcinoma or high-grade dysplasia. Model discrimination was quantified by calculating the area under the receiver operating characteristic curve (C-statistic) with 95\% Cl. Goodnessof-fit was assessed using Hosmer-Lemeshow tests and calibration curves. The analyses were repeated using points categories based on the Parasa et al [4] study (low=0-10 points; intermediate $=>10-20$ points; high $=>20$ points). The cumulative and annual risk of progression to oesophageal adenocarcinoma or high-grade dysplasia were calculated for each category.

The area under the receiver operating characteristic curve when using points categories based on the Parasa et al [4] study was compared to use of low-grade dysplasia only to mimic AGA guidelines, and to use of 3 categories (Short segment non-dysplastic Barrett's oesophagus; long segment non-dysplastic Barrett's oesophagus; and Barrett's oesophagus with low-grade 
dysplasia) to mimic BSG guidelines. Net reclassification index (NRI), was computed as [proportion of all progressors reclassified at higher risk - proportion reclassified at lower risk] - [proportion of all non-progressors reclassified at higher risk - proportion reclassified at lower risk] [9]. The NRIs and proportions in each category, were calculated when changing from a mimic of the BSG guideline system to the points categories proposed by Parasa et al [4].

For comparison with the previous study, multivariate Cox proportional hazards models were used to assess the association between sex, cigarette smoking, Barrett's segment length and low-grade dysplasia status and risk of progression to oesophageal adenocarcinoma or highgrade dysplasia.

To assess potential additions to the model proposed by Parasa et al [4], factors previously associated with risk of progression including age, weight loss, ulceration erosion and gastrooesophageal reflux symptoms [8] were also added to the model individually. If an additional factor was associated with risk of progression $(p<0.05)$, then the C-statistic of the original model plus each additional factor was assessed.

Sensitivity analyses assessed the area under the curve: when outcomes were limited to oesophageal adenocarcinoma only; when points attributed to individual factors were excluded; when Barrett's segment length was reported as short $(<3 \mathrm{~cm}$; 0 points) versus long ( $\geq 3 \mathrm{~cm}$; 5 points, chosen to reflect half the potential maximum 10 points assigned to $10 \mathrm{~cm}$ segment lengths) rather than by centimeter; and when additionally including individuals with missing/unknown data, with missing/unknown data assigned zero points (total $n=3,090$ ).

Analyses were conducted using Stata/IC statistical software (version 14.1, College Station, TX, USA). 


\section{RESULTS}

During 9,487 person-years of follow-up (median 7.5 years follow-up starting 12 months after diagnosis), 54 patients with Barrett's oesophagus progressed to oesophageal adenocarcinoma $(n=41)$ or high-grade dysplasia $(n=13)$.

Applying the point system to our study cohort, we found that the risk of progression to oesophageal adenocarcinoma or high-grade dysplasia increased by $12 \%$ per additional point (HR 1.12, 95\% Cl 1.08-1.16). The model discriminated reasonably well between progressors and non-progressors, with an area under the receiver operating characteristic curve of 0.70 (95\% Cl 0.63-0.78, Table 2). The model was well calibrated according to the calibration slope (C-slope $=1.00)$ and the Hosmer-Lemeshow test $\left(\right.$ chi $^{2}$ statistic $\left.=31.55, P=0.54\right)$.

When categorised into three groups based on points and cut-offs described by Parasa et al [4], relative to the low risk group (annual risk $0.2 \%$ ), risk of progression was higher in the intermediate risk group, (annual risk 0.5\%; HR 2.29, 95\% Cl 0.94-5.59), and the highest risk group (annual risk: 1.5\%; $\mathrm{HR}$ 7.06, 95\% Cl 2.87-17.34) (Figure 2 and 3). Only the latter comparison was statistically significant. Risk of progression in the high risk group was also greater, relative to the intermediate risk group ( $\mathrm{HR} 3.07,95 \% \mathrm{Cl} 1.75-5.43)$. The area under the curve for predicting risk of progression to oesophageal adenocarcinoma or high-grade dysplasia after categorisation was 0.68 (95\% Cl 0.61-0.74, Figure 4). This compares favourably with the area under the curve $(0.70)$ in the original study[4], showing little evidence for overfitting or optimism. A cut-off of $>20$ points (high risk) predicted risk of progression with a sensitivity of $42.6 \%$ and specificity of $84.5 \%$, with an $11.5 \%$ cumulative risk of progression in individuals above this threshold, and only $3.1 \%$ in individuals below this threshold. The intermediate cut-off point $(>10)$ predicted risk of progression with a sensitivity of $88.9 \%$ and 
specificity of $30.4 \%$, with risk of progression of $5.7 \%$ in individuals above this threshold, and only $1.7 \%$ in individuals below this threshold.

\section{Comparison to existing guidelines}

The AUROC for predicting risk of progression to oesophageal adenocarcinoma or high-grade dysplasia using the categories proposed by Parasa et al [4] was improved compared with using low-grade dysplasia status only $(P<0.01, \mathrm{C}=0.58,95 \% \mathrm{Cl} 0.52-0.64)$. There was also some evidence of improvement in discrimination when compared to using the combinations of Barrett's segment length and low-grade dysplasia status recommended by the BSG guidelines $(P=0.08, C=0.61,0.55-0.67)$, although the difference was not statistically significant. In comparison to use of the BSG classification, use of the Parasa et al [4] classification approximately doubled the proportions assigned to the low (12.3\% to $29.6 \%)$ and high risk categories $(8.3 \%$ to $16.7 \%)$ in our sample. The net reclassification index was positive, with $25.9 \%$ of cases and $8.4 \%$ non-cases moving up a risk category and $14.8 \%$ of cases and $26.9 \%$ of controls moving down a risk category. We observed an overall improvement in the net risk stratification of $20.9 \%$ (number of participants reclassified of overall sample was 526 ) for predicting neoplastic progression.

\section{Sensitivity analyses}

None of the additional factors tested changed the AUROC when added individually to the model suggested by Parasa et al [4] (Table 2). In secondary analyses when outcomes were limited to oesophageal adenocarcinoma only, the area under the curve was unchanged, 0.70 (95\% Cl 0.63-0.78, Table 2). Results were largely unchanged when conducting sensitivity analyses assessing model simplification, or inclusion of individuals with unknown/missing data (Table 2). 


\section{DISCUSSION}

In an external validation of a previous risk prediction model [4], we supported previous findings that clinical factors could help to risk stratify patients with Barrett's oesophagus. The risk stratification model predicted risk of progression with an area under the curve of $0.68(95 \% \mathrm{Cl}$ 0.61-0.74), which is in line with the results reported by Parasa et al $(C=0.70)$ [4]. The model also performed better than the use of low-grade dysplasia status alone for risk-stratification, with $20.9 \%$ improvement in the NRI. This may allow tailored endoscopic surveillance and treatment decisions to be made in order to optimise use of endoscopic resources.

Multiple risk models have been derived (and some externally validated) for predicting Barrett's oesophagus [10-14] and oesophageal adenocarcinoma [15-19]. However, few models exist in the setting of risk prediction among patients with Barrett's oesophagus. A recent study used data from 5 centres in the US and 1 Centre in the Netherlands to develop and internally validate a model to predict progression to oesophageal adenocarcinoma or high-grade dysplasia after an initial diagnosis of Barrett's oesophagus [4]. A model including sex, smoking history (ever versus never), Barrett's oesophagus length $(\mathrm{cm})$ and low-grade dysplasia status had reasonable discriminatory ability. The predictive ability of the model proposed by Parasa et al [4], was similar but slightly weaker in the current study cohort than reported previously [4], which is to be expected in external validations. One of the limitations discussed by Parasa et al [4], was that their data relied on patients enrolled at referral academic centres, which may represent higher risk patients. The similarity of our results when using population-based data from the Northern Ireland Barrett's register may be reassuring that the original results are generalizable to broader patient populations. Therefore, this external validation study suggests that the risk prediction model tool could be used for risk-stratification of Barrett's patients following their index endoscopic biopsy to determine surveillance intervals or treatment decisions. 
Our results, using non-US based data from the Northern Ireland Barrett's register, indicate that the categories proposed by Parasa et al [4] may outperform the use of low-grade dysplasia status alone, as recommended by the AGA [1] for risk-stratification purposes. As inter-observer variability is high for judging the presence of low-grade dysplasia [20], the incorporation of other factors may reduce the reliance on low-grade dysplasia for risk stratification purposes. The results also give suggestive evidence that the model proposed by Parasa et al may also outperform the combinations of Barrett's segment length and low-grade dysplasia status recommended by the BSG [2], though the difference was not statistically significant. Whilst the statistical significance remains to be determined, adoption of the Parasa et al categories, could have a significant impact on clinical practice at both ends of the surveillance spectrum. The proportion of individuals assigned to the low and high risk categories both approximately doubled, which could mean more individuals in less frequent surveillance groups (E.g. 3-5 years) but more in the very frequent (6 monthly) surveillance group. Whilst some changes in risk classification were incorrect, none of the changes were major (I.e. highest to lowest or lowest to highest) and a higher proportion of both cases and controls were assigned to a more appropriate risk category. Future health economic models could consider whether the model proposed by Parasa et al [4] is likely to lead to improvements to the cost-effectiveness of surveillance and treatments relative to current guideline recommendations. This may complement efforts to identify biomarkers associated with risk of progression

Changes to guidelines to recommend use of the model proposed by Parasa et al [4] would rely on the collection of smoking data and exact Barrett's segment length, which may hinder application of the full model in some healthcare settings. However, missing data on smoking or other variables may not be problematic as exclusion of smoking or inclusion of missing data (scored as 0 points), which may reflect clinical practice, did not lead to major changes in the predictive ability. Similarly simplification of Barrett's segment length to short versus long, led 
to only minor loss in predictive ability $(0.68,95 \% \mathrm{Cl}: 0.60-0.75)$. Therefore, it is possible that simpler models could be used in cases where smoking information or exact segment length are not available, without compromising the ability to predict risk of progression.

None of the additional factors tested improved risk prediction, despite being associated with the risk of progression previously [8]. Future studies, with longer follow-up time and larger sample sizes, could examine whether additional clinical factors such as age and ulcerative erosion, or biomarkers such as methylation [21,22] or somatic mutations [23-25], could be useful for predicting risk of progression.

Risk models incorporating both genetic and non-genetic factors have previously been developed for predicting risks of various cancers. However, recent studies from our working group and others have found that adding inherited genetic information to a risk model using only clinical and lifestyle factors did not improve discriminatory ability for predicting oesophageal cancer development in the general population.[18,26] Future research should assess whether or not genetic factors or somatic mutations may improve discrimination in the setting of neoplastic progression in Barrett's oesophagus patients.

The current study had several strengths. The Northern Ireland Barrett's register uses population-based data, rather than patients enrolled at referral academic centres that may represent higher risk patients. Thus, we believe our results are not overestimating the results shown. We also had data on the factors studied prior to subsequent diagnosis of oesophageal adenocarcinoma or high-grade dysplasia, so ensuring appropriate temporality of the associations studied.

Limitations of the current study included the smaller sample size than the Parasa et al study [4], particularly as missing data was common due to the use of case note reviews. It is possible that factors included in this model influenced decisions on the frequency of surveillance, which 
may cause reverse causality, whereby individuals with these factors may have more opportunities to detect progression. Unfortunately, information on the number of subsequent endoscopies was not available which prevented an assessment of reverse causality.

In summary, we are the first population-based study to externally validate a risk prediction model to risk stratify patients after an initial diagnosis of Barrett's oesophagus. The model, including sex, dysplasia, smoking history and Barrett's oesophagus segment length, performed well. We also provided novel data demonstrating that this model outperformed current methods such as use of low-grade dysplasia status alone.

\section{REFERENCES}

1 Shaheen NJ, Falk GW, Iyer PG, Gerson LB. ACG Clinical Guideline: Diagnosis and Management of Barrett's Oesophagus. Am J Gastroenterol 2016; 111:30-50.

2 Fitzgerald RC, Di Pietro M, Ragunath K, et al. British Society of Gastroenterology guidelines on the diagnosis and management of Barrett's ooesophagus. Gut 2014;63:742.

3 Gordon LG, Mayne GC, Hirst NG, et al. Cost-effectiveness of endoscopic surveillance of non-dysplastic Barrett's oesophagus. Gastrointest Endosc 2014; 79:242-256.e6.

4 Parasa S, Vennalaganti S, Gaddam S, et al. Development and Validation of a Model to 
Determine Risk of Progression of Barrett's Oesophagus to Neoplasia. Gastroenterology 2018; 154:1282-1289.e2.

5 Thrift AP, Kanwal F, El-Serag HB. Prediction Models for Gastrointestinal and Liver Diseases: Too Many Developed, Too Few Validated. Clin Gastroenterol Hepatol 2016; $14: 1678-1680$.

Bhat S, Coleman HG, Yousef $F$, et al. Risk of malignant progression in Barrett's oesophagus patients: results from a large population-based study. J Natl Cancer Inst 2011; 103:1049-57.

7 Coleman HG, Bhat S, Johnston BT, et al. Tobacco Smoking Increases the Risk of HighGrade Dysplasia and Cancer Among Patients With Barrett's Oesophagus. Gastroenterology 2012; 142:233-240.

8 Coleman HG, Bhat SK, Murray L, et al. Symptoms and Endoscopic Features at Barrett's Oesophagus Diagnosis: Implications for Neoplastic Progression Risk. Am J Gastroenterol $2014 ; 109: 527-534$.

9 Pencina MJ, D'Agostino RB, D'Agostino RB, Vasan RS. Evaluating the added predictive ability of a new marker: From area under the ROC curve to reclassification and beyond. Stat Med 2008; 27:157-172.

10 Thrift AP, Kendall BJ, Pandeya N, Vaughan TL, Whiteman DC. A clinical risk prediction model for Barrett oesophagus. Cancer Prev Res 2012; 5:1115-1123.

11 Thrift AP, Garcia JM, El-Serag HB. A Multibiomarker Risk Score Helps Predict Risk for Barrett's Oesophagus. Clin Gastroenterol Hepatol 2014; 12:1267-1271.

12 Thrift AP, Vaughan TL, Anderson LA, Whiteman DC, El-Serag HB. External Validation of the Michigan Barrett's Oesophagus Prediction Tool. Clin. Gastroenterol. Hepatol. 2017; 
15:1124-1126.

13 Rubenstein JH, Morgenstern $\mathrm{H}$, Appelman $\mathrm{H}$, et al. Prediction of Barrett's Oesophagus Among Men. Am J Gastroenterol 2013; 108:353-362.

14 Liu X, Wong A, Kadri SR, et al. Gastro-Oesophageal Reflux Disease Symptoms and Demographic Factors as a Pre-Screening Tool for Barrett's Oesophagus. PLoS One 2014; 9:e94163.

15 Xie SH, Lagergren J. A model for predicting individuals' absolute risk of oesophageal adenocarcinoma: Moving toward tailored screening and prevention. Int J Cancer 2016; 138:2813-2819.

16 Thrift AP, Kendall BJ, Pandeya N, Whiteman DC. A Model to Determine Absolute Risk for Oesophageal Adenocarcinoma. Clin Gastroenterol Hepatol 2013; 11:138-144.e2.

17 Kunzmann AT, Thrift AP, Cardwell CR, et al. Model for Identifying Individuals at Risk for Oesophageal Adenocarcinoma. Clin Gastroenterol Hepatol Published Online First: 17 March 2018. doi:10.1016/j.cgh.2018.03.014

18 Dong J, Buas MF, Gharahkhani P, et al. Determining Risk of Barrett's Oesophagus and Oesophageal Adenocarcinoma Based on Epidemiologic Factors and Genetic Variants. Gastroenterology 2018; 154:1273-1281.e3.

19 Xie S-H, Ness-Jensen E, Medefelt N, Lagergren J. Assessing the feasibility of targeted screening for oesophageal adenocarcinoma based on individual risk assessment in a population-based cohort study in Norway (The HUNT Study). Am J Gastroenterol 2018; 113:829-835.

20 Vennalaganti P, Kanakadandi V, Goldblum JR, et al. Discordance Among Pathologists in the United States and Europe in Diagnosis of Low-Grade Dysplasia for Patients With 
Barrett's Oesophagus. Gastroenterology 2017; 152:564-570.e4.

21 Alvi MA, Liu X, O'Donovan M, et al. DNA methylation as an adjunct to histopathology to detect prevalent, inconspicuous dysplasia and early-stage neoplasia in Barrett's oesophagus. Clin Cancer Res 2013; 19:878-888.

22 Jin Z, Cheng Y, Gu W, et al. A multicentre, double-blinded validation study of methylation biomarkers for progression prediction in Barrett's oesophagus. Cancer Res 2009; 69:4112-4115.

23 Gregson EM, Bornschein J, Fitzgerald RC. Genetic progression of Barrett's ooesophagus to ooesophageal adenocarcinoma. Br J Cancer 2016; 115:403-410.

24 Bird-Lieberman EL, Dunn JM, Coleman HG, et al. Population-based study reveals new risk-stratification biomarker panel for Barrett's oesophagus. Gastroenterology 2012; 143:927-35.e3

25 Murray L, Sedo A, Scott M, et al. TP53 and progression from Barrett's metaplasia to ooesophageal adenocarcinoma in a UK population cohort. Gut 2006; 55:1390-1397.

26 Kunzmann AT, Canadas Garre M, Thrift AP, et al. Information on Genetic Variants Does Not Increase Identification of Individuals at Risk of Oesophageal Adenocarcinoma Compared to Clinical Risk Factors. Gastroenterology; :doi: 10.1053/j.gastro.2018.09.038. 
Table 1. Baseline characteristics of individuals with Barrett's oesophagus (with specialized intestinal metaplasia) according to progression status to oesophageal adenocarcinoma or highgrade dysplasia.

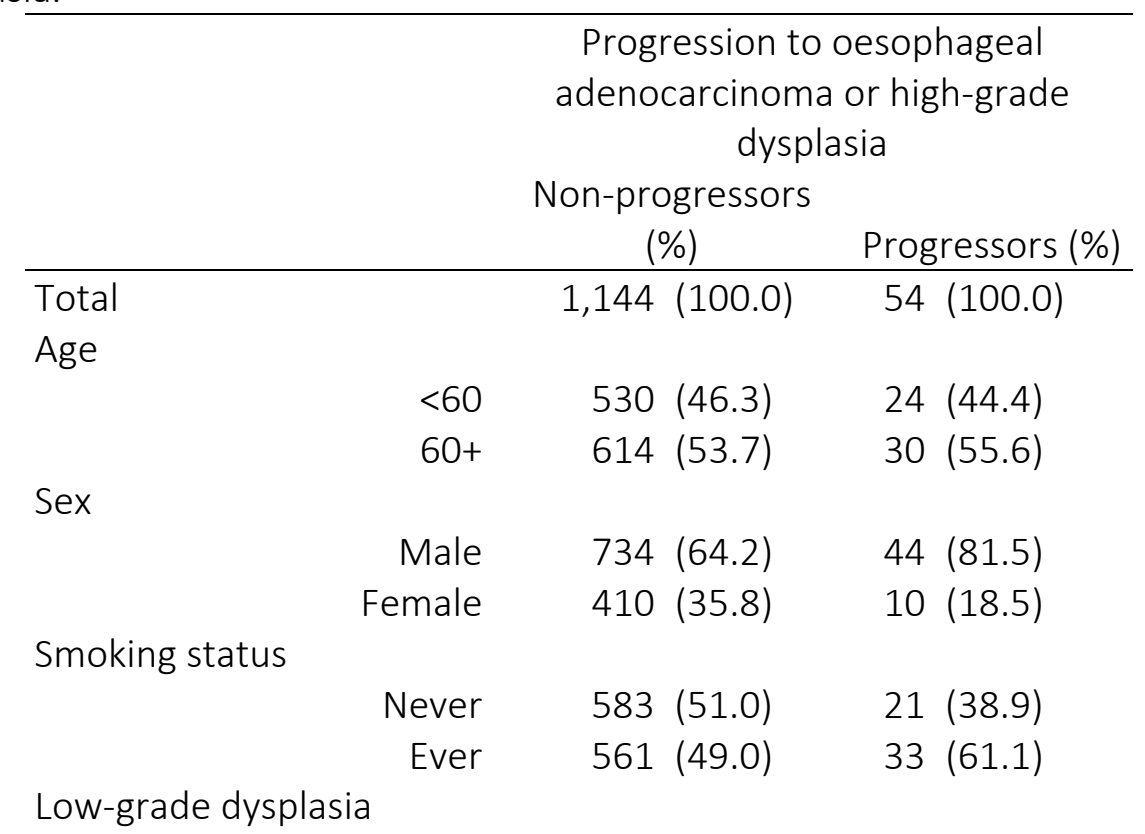




\begin{tabular}{|c|c|c|c|}
\hline No & 1,058 (92.5) & 41 & (75.9) \\
\hline Yes & $86(07.5)$ & 13 & $(24.1)$ \\
\hline Mean Barrett's segment & & 6.7 & \\
\hline length $(\mathrm{cm}, \mathrm{SD})$ & $5.36(2.63)$ & 6 & $(2.55)$ \\
\hline \multicolumn{4}{|l|}{ Reported reflux symptoms } \\
\hline No & $487(42.6)$ & 16 & (29.6) \\
\hline Yes & $657(57.4)$ & 38 & $(70.4)$ \\
\hline \multicolumn{4}{|l|}{ Ulcerative erosion } \\
\hline No & $997(87.2)$ & 41 & (75.9) \\
\hline Yes & $147(12.8)$ & 13 & $(24.1)$ \\
\hline \multicolumn{4}{|l|}{ Weight loss reported } \\
\hline No & $1,012(88.5)$ & 46 & $(85.2)$ \\
\hline \multicolumn{4}{|l|}{ Yes, $<5 \mathrm{~kg}$ or unknown } \\
\hline amount & $63(05.5)$ & 3 & $(05.6)$ \\
\hline Yes, >5kg & $69(06.0)$ & 5 & $(09.3)$ \\
\hline \multicolumn{4}{|l|}{ Risk score } \\
\hline Low & $348(30.4)$ & 6 & $(11.1)$ \\
\hline Intermediate & $619(54.1)$ & 25 & $(46.3)$ \\
\hline High & $177(15.5)$ & 23 & (42.6) \\
\hline
\end{tabular}

Table 2. Area under the receiver operating characteristic curves for predicting risk of progression using points based models proposed by Parasa et al

\begin{tabular}{|c|c|c|c|}
\hline & $\begin{array}{c}\text { Non- } \\
\text { progressors }\end{array}$ & Progressors & AUROC \\
\hline Primary analysis & 1,144 & 54 & $\begin{array}{c}0.70(0.63 \\
0.78)\end{array}$ \\
\hline Risk categories (low, medium, high) & 1,144 & 54 & $\begin{array}{c}0.68(0.61 \\
0.74)\end{array}$ \\
\hline Coefficient based model & 1,144 & 54 & $\begin{array}{c}0.70(0.64 \\
0.78)\end{array}$ \\
\hline \multicolumn{4}{|l|}{ Additional factors } \\
\hline Ulcertative erosion & 1,144 & 54 & $\begin{array}{c}0.71(0.64- \\
0.79)\end{array}$ \\
\hline Reflux symptoms & 1,144 & 54 & $\begin{array}{c}0.71(0.64 \\
0.78)\end{array}$ \\
\hline Weight loss & 1,144 & 54 & $\begin{array}{c}0.71(0.64 \\
0.78)\end{array}$ \\
\hline Age $(<60$ versus $60+)$ & 1,144 & 54 & $\begin{array}{c}0.70(0.62 \\
0.78)\end{array}$ \\
\hline
\end{tabular}


$\underline{\text { Sensitivity analyses }}$

\begin{tabular}{|c|c|c|c|}
\hline Oesophageal adenocarcinoma only & 1,151 & 41 & $\begin{array}{c}0.70(0.63- \\
0.78)\end{array}$ \\
\hline Exclusion of points attributed to smoking & 1,144 & 54 & $\begin{array}{c}0.70(0.63- \\
0.77)\end{array}$ \\
\hline Exclusion of points attributed to sex & 1,144 & 54 & $\begin{array}{c}0.69(0.62- \\
0.77)\end{array}$ \\
\hline Exclusion of points attributed to low-grade dysplasia & 1,144 & 54 & $\begin{array}{c}0.67(0.60- \\
0.75)\end{array}$ \\
\hline $\begin{array}{l}\text { Exclusion of points attributed to Barrett's oesophagus } \\
\text { length }\end{array}$ & 1,144 & 54 & $\begin{array}{l}0.66(0.59- \\
0.74)\end{array}$ \\
\hline Simplification of Barrett's length & & & $\begin{array}{l}0.68(0.60- \\
0.75)\end{array}$ \\
\hline Inclusion of missing data as 0 points & 2,976 & 114 & $\begin{array}{c}0.68(0.62- \\
0.73)\end{array}$ \\
\hline
\end{tabular}

\section{FIGURE LEGENDS:}

Figure 1. Selection of participants for primary analysis with complete data

Figure 2. Total risk of progression to oesophageal adenocarcinoma or high-grade dysplasia by risk category groups proposed by Parasa et al.

${ }^{1}$ Proportion in each risk category group progressing during a median 7.5 years of follow-up.

${ }^{2}$ Annual risk of progression in each risk category group in parentheses.

Figure 3. Cumulative incidence of progression to oesophageal adenocarcinoma or high-grade dysplasia by risk category groups proposed by Parasa et al. 
Figure 4. Area under the receiver operating characteristic curve for predicting risk of progression to oesophageal adenocarcinoma or high-grade dysplasia when using risk category groups proposed by Parasa et al. 
STROBE Statement-checklist of items that should be included in reports of observational studies

\begin{tabular}{|c|c|c|c|c|}
\hline & $\begin{array}{l}\text { Item } \\
\text { No. }\end{array}$ & Recommendation & $\begin{array}{l}\text { Page } \\
\text { No. }\end{array}$ & $\begin{array}{l}\text { Relevant text } \\
\text { from manuscript }\end{array}$ \\
\hline \multirow[t]{2}{*}{ Title and abstract } & 1 & $\begin{array}{l}\text { (a) Indicate the study's design with a commonly used term in the title or the } \\
\text { abstract }\end{array}$ & 1 & \\
\hline & & $\begin{array}{l}\text { (b) Provide in the abstract an informative and balanced summary of what was } \\
\text { done and what was found }\end{array}$ & 3 & \\
\hline \multicolumn{5}{|l|}{ Introduction } \\
\hline Background/rationale & 2 & $\begin{array}{l}\text { Explain the scientific background and rationale for the investigation being } \\
\text { reported }\end{array}$ & 4 & \\
\hline Objectives & 3 & State specific objectives, including any prespecified hypotheses & 5 & \\
\hline \multicolumn{5}{|l|}{ Methods } \\
\hline Study design & 4 & Present key elements of study design early in the paper & 6 & \\
\hline Setting & 5 & $\begin{array}{l}\text { Describe the setting, locations, and relevant dates, including periods of } \\
\text { recruitment, exposure, follow-up, and data collection }\end{array}$ & 6 & \\
\hline Participants & 6 & $\begin{array}{l}\text { (a) Cohort study-Give the eligibility criteria, and the sources and methods of } \\
\text { selection of participants. Describe methods of follow-up } \\
\text { Case-control study-Give the eligibility criteria, and the sources and methods } \\
\text { of case ascertainment and control selection. Give the rationale for the choice } \\
\text { of cases and controls }\end{array}$ & 7 & \\
\hline
\end{tabular}




\begin{tabular}{|c|c|c|c|}
\hline & & $\begin{array}{l}\text { Cross-sectional study-Give the eligibility criteria, and the sources and } \\
\text { methods of selection of participants }\end{array}$ & \\
\hline & & $\begin{array}{l}\text { (b) Cohort study-For matched studies, give matching criteria and number of } \\
\text { exposed and unexposed } \\
\text { Case-control study-For matched studies, give matching criteria and the } \\
\text { number of controls per case }\end{array}$ & \\
\hline Variables & 7 & $\begin{array}{l}\text { Clearly define all outcomes, exposures, predictors, potential confounders, and } \\
\text { effect modifiers. Give diagnostic criteria, if applicable }\end{array}$ & 6 \\
\hline $\begin{array}{l}\text { Data sources/ } \\
\text { measurement }\end{array}$ & $8^{*}$ & $\begin{array}{l}\text { For each variable of interest, give sources of data and details of methods of } \\
\text { assessment (measurement). Describe comparability of assessment methods } \\
\text { if there is more than one group }\end{array}$ & 6 \\
\hline Bias & 9 & Describe any efforts to address potential sources of bias & \\
\hline Study size & 10 & Explain how the study size was arrived at & $\begin{array}{lr}7 & \& \\
\text { Figure } 1\end{array}$ \\
\hline
\end{tabular}

Continued on next page 


\begin{tabular}{|c|c|c|c|}
\hline $\begin{array}{l}\text { Quantitative } \\
\text { variables }\end{array}$ & 11 & $\begin{array}{l}\text { Explain how quantitative variables were handled in the analyses. If applicable, describe which } \\
\text { groupings were chosen and why }\end{array}$ & $7-8$ \\
\hline \multirow{7}{*}{$\begin{array}{l}\text { Statistical } \\
\text { methods }\end{array}$} & \multirow[t]{7}{*}{12} & (a) Describe all statistical methods, including those used to control for confounding & $7-8$ \\
\hline & & (b) Describe any methods used to examine subgroups and interactions & $7-8$ \\
\hline & & (c) Explain how missing data were addressed & $7-8$ \\
\hline & & (d) Cohort study-If applicable, explain how loss to follow-up was addressed & $7-8$ \\
\hline & & Case-control study_-If applicable, explain how matching of cases and controls was addressed & \\
\hline & & Cross-sectional study-If applicable, describe analytical methods taking account of sampling strategy & \\
\hline & & (e) Describe any sensitivity analyses & $7-8$ \\
\hline \multicolumn{4}{|l|}{ Results } \\
\hline \multirow[t]{3}{*}{ Participants } & \multirow[t]{3}{*}{$13^{*}$} & $\begin{array}{l}\text { (a) Report numbers of individuals at each stage of study-eg numbers potentially eligible, examined } \\
\text { for eligibility, confirmed eligible, included in the study, completing follow-up, and analysed }\end{array}$ & 9 \\
\hline & & (b) Give reasons for non-participation at each stage & \\
\hline & & (c) Consider use of a flow diagram & Fig 1 \\
\hline \multirow[t]{3}{*}{ Descriptive data } & \multirow[t]{3}{*}{$14^{*}$} & $\begin{array}{l}\text { (a) Give characteristics of study participants (eg demographic, clinical, social) and information on } \\
\text { exposures and potential confounders }\end{array}$ & Table 1 \\
\hline & & (b) Indicate number of participants with missing data for each variable of interest & $\begin{array}{l}\text { Complete } \\
\text { case }\end{array}$ \\
\hline & & (c) Cohort study-Summarise follow-up time (eg, average and total amount) & Table 1 \\
\hline Outcome data & $15^{*}$ & Cohort study-Report numbers of outcome events or summary measures over time & Table 2 \\
\hline
\end{tabular}


Case-control study-Report numbers in each exposure category, or summary measures of exposure

Cross-sectional study-Report numbers of outcome events or summary measures

Main results

16 (a) Give unadjusted estimates and, if applicable, confounder-adjusted estimates and their precision

(eg, 95\% confidence interval). Make clear which confounders were adjusted for and why they were included

(b) Report category boundaries when continuous variables were categorized

(c) If relevant, consider translating estimates of relative risk into absolute risk for a meaningful time period

Continued on next page 


\begin{tabular}{|c|c|c|c|}
\hline \multicolumn{4}{|l|}{ Discussion } \\
\hline Key results & 18 & Summarise key results with reference to study objectives & 11 \\
\hline Limitations & 19 & $\begin{array}{l}\text { Discuss limitations of the study, taking into account sources of potential bias or imprecision. Discuss both direction } \\
\text { and magnitude of any potential bias }\end{array}$ & 14 \\
\hline Interpretation & 20 & $\begin{array}{l}\text { Give a cautious overall interpretation of results considering objectives, limitations, multiplicity of analyses, results } \\
\text { from similar studies, and other relevant evidence }\end{array}$ & 14 \\
\hline Generalisability & 21 & Discuss the generalisability (external validity) of the study results & 14 \\
\hline \multicolumn{4}{|c|}{ Other information } \\
\hline Funding & 22 & $\begin{array}{l}\text { Give the source of funding and the role of the funders for the present study and, if applicable, for the original study } \\
\text { on which the present article is based }\end{array}$ & 1 \\
\hline
\end{tabular}

